\title{
Performance Evaluation of CMMBCR Method to Improve Battery Power Awareness in Ad Hoc Network
}

\author{
Anmol S. Suryavanshi ${ }^{1}$, Nitesh Rastogi ${ }^{2}$ \\ PG Student, Dept. Of CSE, M.P, India ${ }^{1}$
}

Assistant professor, Dept. of CSE, JDCT, Indore, M.P, India ${ }^{2}$

\begin{abstract}
MANET is collection of mobile nodes that form a network independently of any centralized administration. Since those mobile devices are battery operation based and extending the battery lifetime has become an main aim. Generally all the researchers have recently started to consider power-aware development of efficient protocols for MANETs. When each mobile node in a MANETs performs the routing function for effective establishment of communication among different mobile nodes the "Death" of even an any of the nodes due to power exhaustion might cause disconnect of services in the entire MANETs. So, mobile nodes in MANETs are battery based. Thus, they affect from limited energy level problems. When the nodes in the network are moving if a node moves out of a radio range of the other node then link between them is broken. Mainly due to this environment there are two major conditions of a link break that is Node dead due to energy collapse and Node going out of the radio range of its adjacent node [1].
\end{abstract}

KEYWORDS: MANET; AODV, IDS, Performance Factors, CMMBCR (Conditional Max-Min Battery Capacity Routing);

\section{I.INTRODUCTION}

Several methods have been formed to address the energy efficiency issues in Ad-Hoc Networks. These techniques are different in the methodology as well as in the layer of the protocol stack at which they perform their job. Algorithms belong to the group which may operate at the MAC layer, Network layer, or in between them. This following section mainly covers the most significant algorithms that belong to these categories. [2] Power-Aware Routing explore poweraware metrics to use with Routing Protocols on top of their MAC power savings protocol, PAMAS. They indicate that the strategy followed by the different Routing Protocols that are not power conscious would lead to fast depletion of battery power and hence quick degradation of the Network operation. We have already discussed these metrics. This mechanism implemented the first and fourth metrics (to minimize energy frenzied per packet and minimize cost per packet, respectively). In this modeling, it used inadequately populated networks and they did not judge mobility in their simulations. The main reason behind not using mobility is that the assessment is done for power controlling and not routing, this mechanism shows mobility has a considerable effect on the performance of power efficient mechanisms.[3]

Maximum Battery Life Routing- A power-aware Routing Protocol that distributes power consumption evenly over nodes and minimizes the overall transmission power is proposed in .It uses the protocol for conditional max-min battery capacity routing (CMMBCR) scheme. When all nodes on some possible routes between a source and a destination have sufficient remaining energy above a certain value, the route with the minimum total transmission power (MTRP) among these routes is chosen[4]. If all paths have nodes with very low battery capacity, the routes that include nodes with the least battery power should be avoided to extend the life span of the nodes. If value of $\gamma$ is zero, the CMMCBR reduces to MTRP. If the value of $\gamma$ is equal to the maximum (100), the CMMBCR scheme reduces to the Min-Max battery cost routing (MMBCR) scheme. [5]. 


\title{
8 \\ ISSN (Print) : $2320-3765$ \\ ISSN (Online): 2278 - 8875 \\ International Journal of Advanced Research in Electrical, Electronics and Instrumentation Engineering
}

\author{
(An ISO 3297: 2007 Certified Organization)
}

\section{Vol. 3, Issue 8, August 2014}

Analysis of Energy Efficient Routing Techniques- The main focus of research on Routing Protocols in MANETs has been Network performance. There has been some study on Energy aware Routing Protocols for mobile Ad hoc network which is used in this mechanism.

Minimum Energy Routing- Mechanism proposes a routing algorithm based on minimizing the amount of energy per bit required to get a packet from source to destination [6].

\section{LITERATURE SURVEY}

When we get idea from different research material we get a uniform approach for minimize battery failure or node lose problem, for proper result orientation we get related data from different topics. First a metrics used by conventional routing protocols for the wired Internet, which is oblivious to an energy budget, typically do not need to consider any energy-related parameters. Thus, RIP uses hop count as the sole route quality metric, thereby, selecting minimum-hop paths between the source and destinations. OSPF, on the other hand, supports additional link metrics such as available bandwidth, link propagation delay etc. These algorithms, however, may result in a rapid depletion of the battery energy in the nodes along the most heavily-used paths in the network. Routing protocols for wireless ad hoc environments contain special features to reduce the signalling overheads and convergence problems caused by node mobility and potential link failures.

When any protocol do not necessarily compute the absolute minimum-cost path, they aim at selecting paths that have lower cost (in terms of metrics such as hop count or delay). Such protocols must be modified to yield energy-efficient routing solutions.A large number of researchers has addressed the problem of energy efficient data transfer in the context of multi-hop wireless networks. Existing protocols may be classified into two distinct categories. One category of protocols is based on minimum-power routing algorithms, which focus on minimizing the power requirements over end-to-end paths. A typical protocol in this category selects a routing path from a source to some destination so as to minimize the total energy consumption for transmitting a fixed number of packets over that path. Each link cost is set to the energy required for transmitting one packet of data across that link and Dijkstra's shortest path algorithm is used to find the path with the minimum total energy consumption. These protocols traditionally ignore the power dissipated on the receiver side in a node, and therefore, tend to result in routing paths with a large number of short hops. A key disadvantage of these protocols is that they repeatedly select the least-power cost routes between source-destination pairs. As a result, nodes along these least-power cost routes tend to "die" soon by rapidly exhausting their battery energy. This is doubly harmful since the nodes that die early are precisely the ones that are most needed to maintain the network connectivity [7]

This revolution began with Albrecht's introduction of Function Points. And although other works were going in this way, not any did so with the pragmatism and simplicity. Function Points were then developed over time, becoming an object of consistency, with a group of international interest: the International Function Point Users Group. That was happened to Function Points which, after the advent of Charles R. Symons, underwent the schism that was given in this work; we shall consider only Function Point metrics since they are the most widespread throughout the world. So, why are functional metrics of use? Since they meet at least two fundamental needs experienced by organizations:

1. Measuring software on a "business value" basis from the user's point of view.

2. Obtaining a measurement as independent as possible from the productive contexts in which the same application is developed and utilized [8].

\section{PROBLEM STATEMENT}

The nodes in an ad hoc network are constrained by battery power for their operation. To route a packet from a source to a destination involves a sufficient number of intermediary nodes. Hence, the precious resource of a node is a battery power that must be used efficiently in order to avoid early termination of a node or a network. Thus, an important issue is energy aware routing in such networks. The major means of increasing the life of a node is Power aware routing. The lifetime of a network is usually defined according to the following criteria:

Fig. 1. The time until the first node burns out its entire battery budget;

Fig. 2. The time until a certain proportion of the nodes fails; and

Fig. 3. The time until network partitioning occurs. 


\title{
10 Electronics and Instrumentation Engineering
}

\author{
(An ISO 3297: 2007 Certified Organization)
}

\section{Vol. 3, Issue 8, August 2014}

The communication related power consumption is mainly due to transmit-receive module present in the nodes. Whenever a node leftovers active, that is, energy gets consumed during transmission or reception of a packet. Even when the node is not actively participating in communication but is in the listening mode (waiting for the packets), the battery keeps discharge. The computation power refers to the power spent in calculations that take place in the nodes during routing and power adjustment $[1,9]$.

Thus all the above problems are unsolved and open the area of work for various researchers. Out of those this work is getting its concern deeper about designing the updated HCF mechanism which is lighter in computational load and size. The suggested approach will improves the quality of service of the network by minimizing the number of false positives.

\section{PROPOSED CMMBCR APPROACH}

The objective of proposed work is improve transmission periods of nodes via specifying minimum residual energy level of nodes to prevent data transmission from uneven manner. The projected approach also offered to sending prioritized data packets and discard Route Request packets comes from other nodes during regular communication. This work minimized battery dissipation via discarding route discovery request packets and maximize the period of data transmission. The whole working of the approach is defined in below steps and work flow.

Step1- firstly nodes are charged with battery power $\{\mathrm{E}=100\}$

Step2-Then set two threshold values for prioritize data transmission and alerting $\{$ TV1=25 and TV2=1 $\}$

Step3-Verify battery power of nodes and taken some actions during the routing procedures.

If battery power is much than first threshold value $\{\mathrm{E}>\mathrm{TV} 1\}$ then nodes send regular data packets.

In addition if battery power is less than equal to first threshold value and much than second threshold value $\{\mathrm{E}<=25$ and $E>1\}$ then nodes send prioritize data packets and discard route request packets from other nodes.

Flow Diagram of proposed approach

In this proposed work a novel mechanism is proposed which is used to identify the spoofed packet out of numerous rightful packets. It has four components:
(i) Source Node
(ii) Destination Node
(iii) Network

The proposed approach is capable of identifying the spoofed packet out of larger number of normal packets. Now the task is to improve the correctness of approach. After that various experiments is been performed on which regular results is generating. At the primary level of this research the approach seems to provide better results than any lexisting approaches.

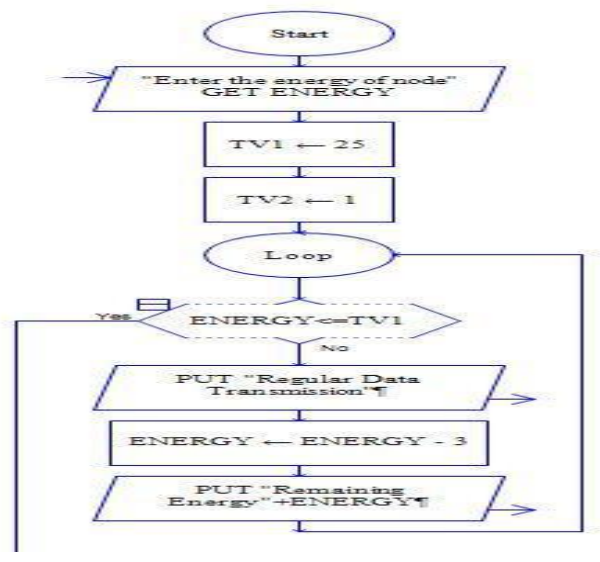

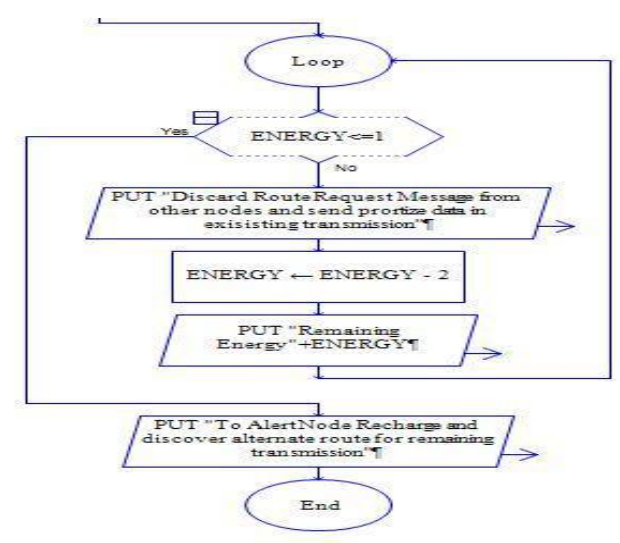




\section{2 \\ ISSN (Print) : $2320-3765$ \\ ISSN (Online): 2278 - 8875 \\ International Journal of Advanced Research in Electrical, Electronics and Instrumentation Engineering \\ (An ISO 3297: 2007 Certified Organization)}

Vol. 3, Issue 8, August 2014

Work-Flow of Proposed Approach

Proposed Algorithms

Proposed Connection Establishment and Route Update Algorithm \& with Threshold Value and Minimum Threshold value.

Initial energy $=\mathrm{E} \quad($ Suppose $\mathrm{E}=100)$

Threshold energy $=\Theta_{1}(20 \%$ of $\mathrm{X})$ //for alert the energy level.

Minimum threshold energy $=\Theta_{2}(5 \%$ of $E) / /$ for recharge

1) If $\left\{\left(E>\Theta_{1}\right) \& \&\right.$ (radio range from source to next hop $\left.<250 \mathrm{~m}\right) \& \&$ (next hop == destination) $\}$

Then

\{Establish connection from source to destination\}

Else

2) If $\left\{\left(\mathrm{E} \leq \Theta_{1}\right)\right\}$

\{Nodes will broadcast the message in network only Important packets are forward \& those who already Establish connection transfer their data before energy Level reaches to minimum threshold level \}

3) If $\left\{\left(\mathrm{E}==\mathrm{\Theta}_{2}\right)\right\}$

\{Node will be in charging mode \& route will update

4) If $\{$ (node energy charge up $==100) \& \&$ ( radio range

From source to next hop $\leq 250 \mathrm{~m})\}$

\{Go to step 1$\}$

End

Description: The main goals of the algorithm are fair energy conservation via:

[1] Rotating sleep periods equally among network nodes thus giving nodes equal opportunity for reducing energy consumption

[2] Supporting routing algorithms in making routing decisions based on energy fairness

[3] Little impact on network operation, for example, introduces slight or no additional traffic or energy cost.

From a functional point of view, proposed algorithm can be considered to consist of two main units. One unit from these handles the energy conservation operation. This is done through managing the nodes' energy level periods. The other aspect of the algorithm, as far as energy management decisions are concerned, takes care of supporting the routing protocol. It helps to ensure the routing protocol makes routing decisions that serve a specific goal. In this for example, whenever possible, nodes carry out routing duties that are proportional to their energy levels compared to each other. $[1,10]$

Now AODV generate a RREQ message to their neighbours if node energy is greater than threshold value then establish connection and forwarded request up to destination. Then all the nodes send back the RREP message to their sender according to energy condition criteria.

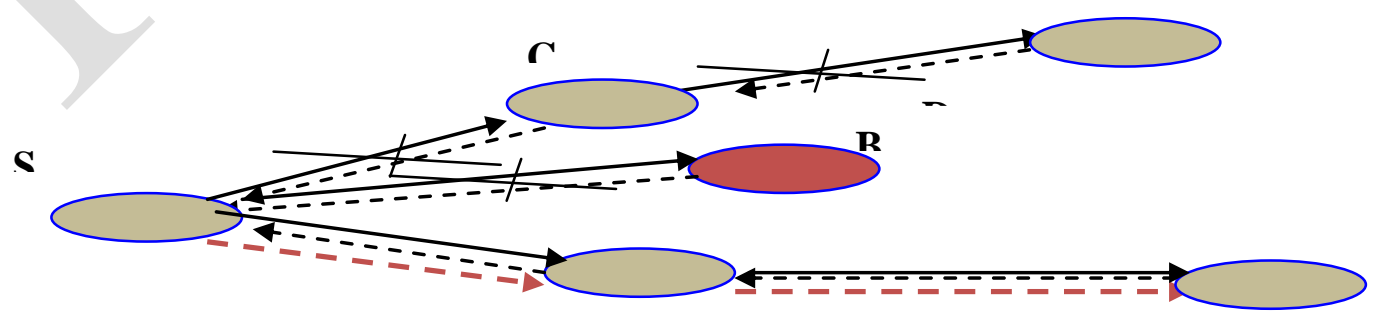

A

$\mathbf{E}$

Fig. 1.1 Energy based connection establishment

10.15662/ijareeie.2014.0308015 
 Electronics and Instrumentation Engineering}

\section{(An ISO 3297: 2007 Certified Organization)}

\section{Vol. 3, Issue 8, August 2014}

The red colour nodes in the network are low level energy nodes so no connection will establish according to proposed algorithm. The numbers of nodes in the network are only take part in communication if they have a sufficient energy except the important packet condition. Now in a given simulation time if all the nodes are reaches to threshold level then in that case (if required) the RREQ and RREP procedure are calling for forwarding important packets in the network between two energy levels. [1]

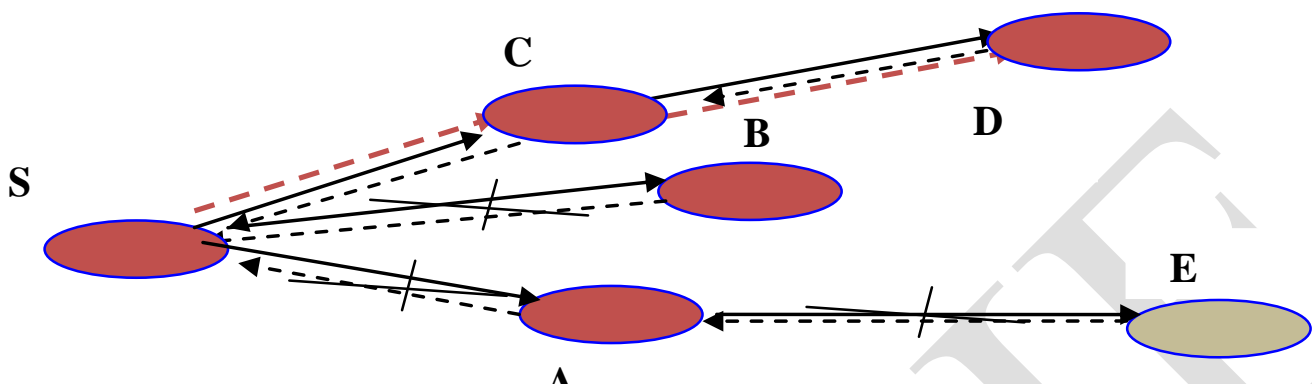

A

Fig.1.2 Routing with Important Packets

Now if the energy level of maximum number of nodes are in between threshold and minimum threshold value then same RREQ and RREP route discovery procedure will call till energy reaches to minimum threshold value. Here only the communication is possible among low level energy nodes. Now if the energy of nodes is reaches to minimum threshold value then in that case the nodes are not take part in communication and route will update.

\section{RESULT EVALUATION}

In future the results will show the effectiveness of proposed scheme. For network simulation, there are several performance metrics which is used to evaluate the performance. In future simulation purpose this work will use different performance metrics for showing the expected results. Results are plotted using Xgraph utility of NS2

Packet delivery ratio $(P D R)$ - It is the ratio of number of packets received at the destination to the number of packets sent from the source. When packet delivery ratio is high the performance is better.

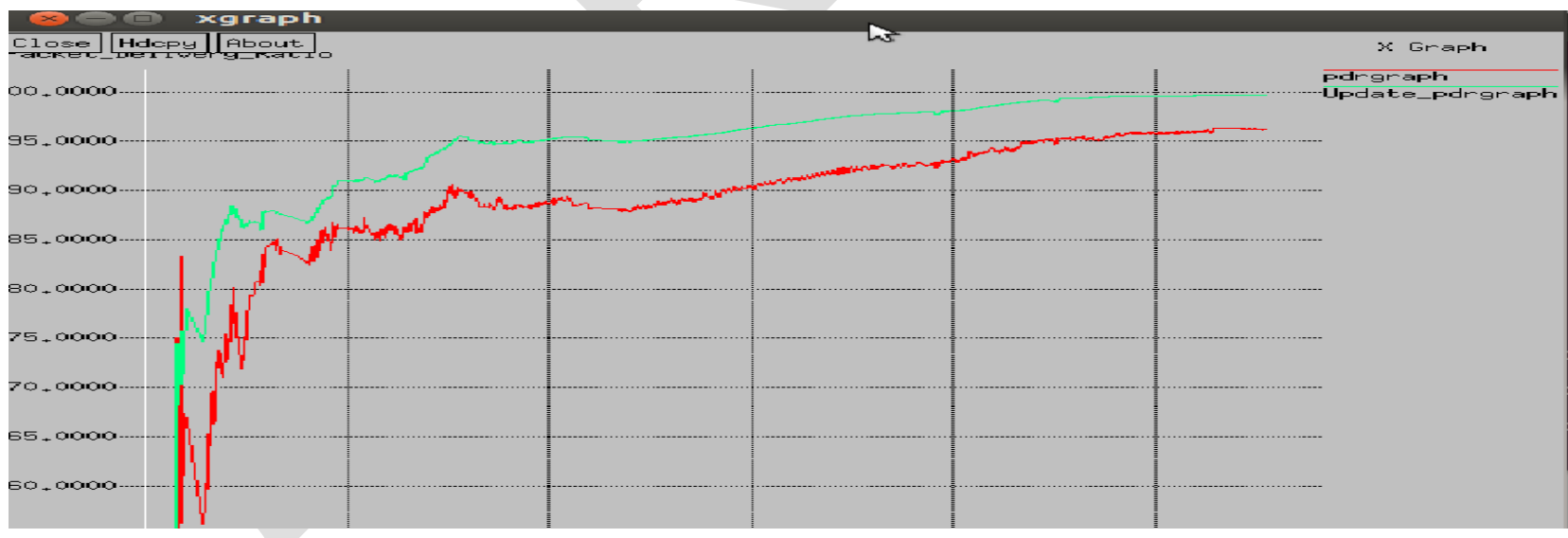

Graph 1: Comparison of PDR Ratio of CMMBCR Proposed and Existing

Graph Summary: As the PDR ratio is used to identify the performance of the approaches using the packet delivery ration. It is the ration of number of packet sent to the number of packet received. In ideal condition it should be high as possible. For comparing the suggested work of CMMBCR, the above graph interprets the result as an improved PDR ration than the existing approaches. 


\section{International Journal of Advanced Research in Electrical, Electronics and Instrumentation Engineering}

\section{(An ISO 3297: 2007 Certified Organization)}

\section{Vol. 3, Issue 8, August 2014}

Throughput-It is one of the dimensional parameters of the network which gives the fraction of the channel capacity used for useful transmission selects a destination at the beginning of the simulation i.e., the information whether or not data packets correctly delivered to the destinations.

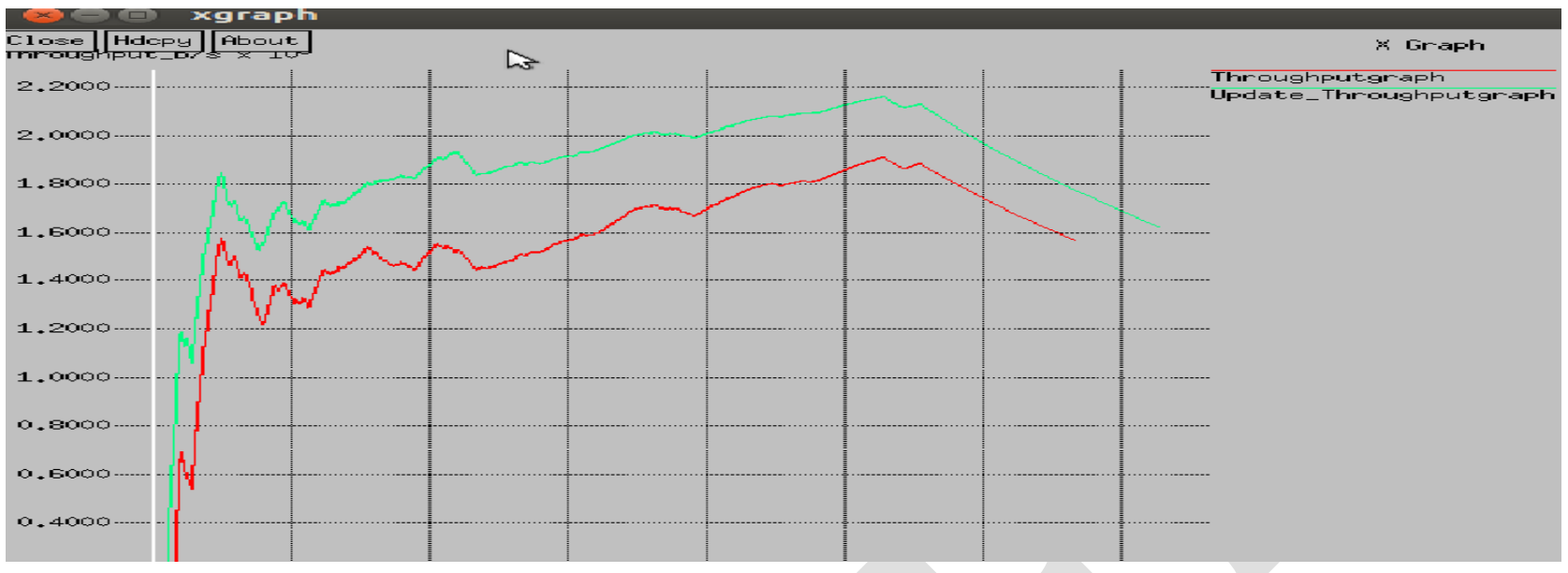

Graph 2: Comparison of Throughput of CMMBCR Proposed and Existing Throughput

Graph Summary: As throughput measure the transmission efficiency in terms of successfully delivered packets in unit time for a specified channel bandwidth. The above graph shows the effectiveness of the suggested approach while comparing it with the existing. The graph interprets the constant throughput for several cases which justify the approach.

Routing Overhead: Routing Load is the ratio of total number of the routing packets to the total number of received data packets at destination. The amount of battery consumed generated (in bits) per data traffic delivered (in bits). It should be taken in terms of the extra load started while executing the suggested approach than the normal protocol load for the system. Considering the factors

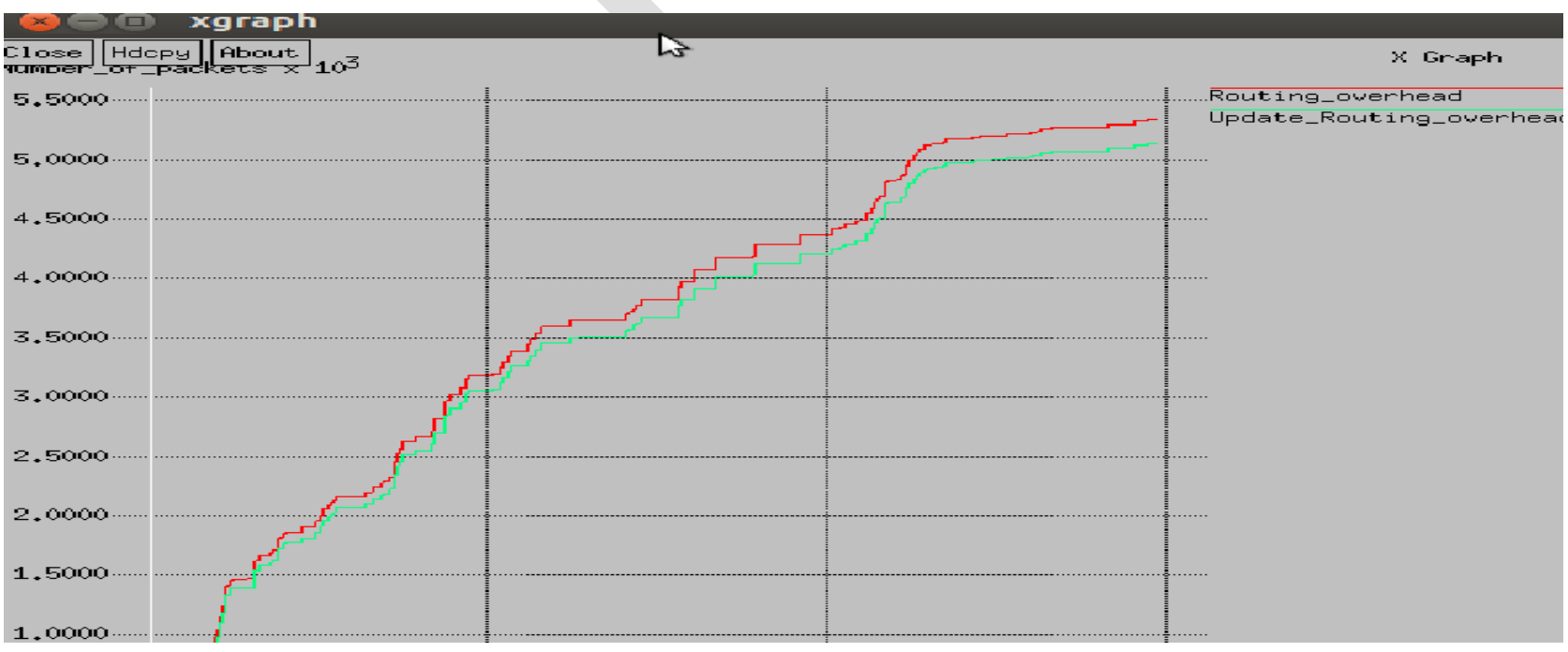

Graph 3: Comparison of Routing Overhead of CMMBCR Proposed and Existing Overhead

Graph Summary: The above graph verifies its results by minimum routing overhead associated with the suggested approach. It also shows that the complexity of using the proposed method is quite less in comparison with the existing. 


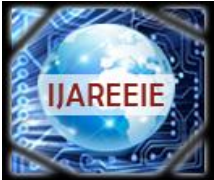 \\ ISSN (Print) : $2320-3765$ \\ ISSN (Online): $2278-8875$ \\ International Journal of Advanced Research in Electrical, Electronics and Instrumentation Engineering
}

\author{
(An ISO 3297: 2007 Certified Organization)
}

\section{Vol. 3, Issue 8, August 2014}

\section{VI.CONCLUSION}

This scheme has been utilizes power status of each mobile node and alternate paths. This scheme can be incorporated into any ad hoc on-demand routing protocol to improve reliable packet delivery in the face of node movements and route breaks. Alternate routes are utilized only when data packets cannot be delivered through the primary route. As a case study, it has been applied to AODV and performance has been studied via simulations. Simulation results have indicated that new technique provides robustness to mobility and enhances protocol performance. However, this scheme may not perform well under sparse traffic networks. Its performance has been found much better than other existing protocols in dense medium as probability of finding active routes increases.According to our simulation result we also get important packet transmission concept. This one is the one unique concept to utilize energy remaining after threshold level.[11,12]

However, field trials and experiments with existing MANETs prove that the performance of MANETs is still far below what they are expected to be. As explained throughout this dissertation, many open research issues need to be resolved:

- Scalability. Based on existing CMMBCR scheme, the network performance, indexed by throughput, end-to-end delay, and fairness, is not scalable with either the number of nodes or the number of hops in the network. [13,14]

- Self-organization and self-configuration. Self organization and self-configuration require all protocols in MANETs to be distributive and collaborative. Otherwise, MANETs will lose the autonomic feature. However, current MANETs can only partially realize this objective.

- Security. Due to wireless ad hoc architecture, MANETs not prevented the battery lose problem uniformly so that this technique is very useful to achieve the results.[15]

\section{FUTURE WORK}

Some problems and concepts that remain unaddressed can be performed in future. This system can further be extended to implement CMMBCR scheme in real-time networks where it has to deal with real-time requests. Such as with the help of pre-emptive approach more information can be added for exact timely analysis node dropping minimization problem can easily solved, CMMBCR scheme provide lower overheads with high accuracy. We are also working towards embedding the developing source code of our proposed scheme in NS2. In our proposed scheme so as to use the benefits of approach like open source.

\section{ACKNOWLEDGMENT}

This research work is self financed but recommended from the institute so as to improve the battery power awareness with current techniques in Ad Hoc network using CMMBCR. Thus, the authors thank the anonymous reviewers for their valuable comments, which strengthened the paper. The authors also wish to acknowledge institute administration for their support \& motivation during this research.

\section{REFERENCES}

[1] Anmol Suryavanshi et al., International Journal of Advanced Research in Computer Science and Software Engineering 4(3), March - 2014, pp. 755-760

[2] Forman G., Zahorjan J. The challenges of mobile computing. IEEE Computer 1994; 27(4):38-47.

[3] Perkins C. Ad Hoc Networking: Addison-Wesley: 2001; 1-28.

[4] N. Vassileva, F. Barcelo, "A Survey of Routing Protocols for Maximizing the Lifetime of Ad Hoc.

[5] Wireless Networks,” International Journal of Software Engineering and Its Applications, Vol. 2, No. 3, pp. 77-89, 2008

[6] S. Singh, M. Woo, C. Raghavendra, "Power-aware Routing in Mobile Ad Hoc Networks," Proceedings of the ACM Mobile Computing and Networking Conference, Dallas, Texas, pp. 181-190, 1998

[7] C. Toh, "Maximum Battery Life Routing to Support Ubiquitous Mobile Computing in Wireless Ad Hoc Networks," IEEE Communications Magazine, pp. 2-11, 2001

[8] L. M. Feeney and M. Nilsson, "Investigating the Energy Consumption of a Wireless Network Interface in an Ad Hoc Networking Environment," Proceedings of IEEE INFOCOM 2001, Vol. 3, Anchorage AK, pp. 1548-1557, 2001

[9] V. Rishiwal, M. Yadav, S. Verma, S. K. Bajapai, "Power Aware Routing in Ad Hoc Wireless Networks, Journal of Computer Science and Technology," Vol. 9, No. 2, pp. 101-109, 2009 


\section{International Journal of Advanced Research in Electrical,} Electronics and Instrumentation Engineering

\section{(An ISO 3297: 2007 Certified Organization)}

\section{Vol. 3, Issue 8, August 2014}

[10] R. Kravets and P. Krishnan, "Power Management Techniques for Mobile Communications", Proceedings of the ACM Mobile Computing and Networking Conference, Dallas, Texas, October 1998, pages 157-168.

[11] Li Q, AslamJ, Rus D. Online Power-aware Routing inWireless Ad-hoc Networks. Proceedings of Int'l Conf. on Mobile Computing and Networking (MobiCom'2001) 2001.

[12] Perkins C, Bhagwat P. Highly Dynamic Destination-Sequenced Distance-Vector Routing (DSDV) for Mobile Computers. Computer Communications Review 1994; 234-244.

[13] Pei G, Gerla M, Chen T-W. Fisheye State Routing: A Routing Scheme for Ad Hoc Wireless Networks. Proceedings of IEEE Int'l Conf. on Communications (ICC) 2000; 70-74.

[14] Johnson D, Maltz D. Dynamic Source Routing in ad hoc wireless networks. Mobile Computing (edited by Imielinski T, Korth H); Kluwer Academic, 1996; 153-181. 